\title{
Towards a Social Semiotic Approach of the Analysis of Emotion in Sound and Music
}

\author{
David Machin, \\ Cardiff School of Journalism, Media and Cultural Studies, Cardiff University \\ Cardiff, United Kingdom
}

\begin{abstract}
This paper explores the possibility of a social semiotic approach to popular music in order to document the voice qualities, melodies and instrumental sounds found in popular music using the case study of Michael Jackson's 'Billie Jean'. The paper explores how these semiotic features, as well as the lyrics, communicate and reveal something of the subjectivity, the emotional world, created by musicians. Here the analysis explores Jackson's use of restricted pitch ranges, breathiness, melodic patterns and gentle disjunctive articulation showing how these help to communicate the 'tension', and 'trouble' in the song. Taking a social semiotic approach the paper identifies the underlying available repertoire of meanings available to musicians and shows how musicians can skilfully draws upon these.
\end{abstract}

\section{Introduction}

Michael Jackson's Thriller album was originally reviewed using used words such as 'slick', 'harrowing', 'dark messages', 'tense and obsessive sound'. Connolly, writing in Rolling Stone (1983), commented that the record has 'a deeper, if less visceral, emotional urgency than any of his previous work'. The conclusion was that the words and sounds of the album revealed something about the changing identity of the troubled genius of Jackson pointing to his lack of fit to society and troubled family relationships. Yet such commentaries make observations through vaguer adjectives such as 'tension' and 'obsessive' rather than telling us exactly what it is in the music that communicates these meanings. There has been much less attention too in popular music studies, with some notable exceptions (Tagg, 1982; Middleton, 1996; Walser, 1993) to the way that sounds themselves communicate meanings. While we can of course carry out analyses of lyrics it is how these are delivered, the melodies and sound qualities that carry them, along with the sounds of instruments and drums, which also forms the way they convey moods, ideas and subjectivities. As regards Jackson's 'Billie Jean' the meaning of the words, the story about an obsessed female fan, lies also in the sounds. In this paper, taking a social semiotic approach, the aim is to reveal something of the subjectivity, the emotional world, created by Michael Jackson in his music through a systematic analysis of his use of a number of semiotic resources: restricted pitch ranges, 
breathiness, and gentle disjunctive articulation, and instrumentation showing how these point to 'tension', 'obsession', 'troubled relationships' and 'emotional urgency'.

A social semiotic approach is interested in the way that communicators use semiotic resources to achieve particular goals, to communicate specific ideas, attitudes, values and identities. It is interested in exploring on the one hand the resources available to communicators, in other words the repertoire of sign/meaning potentials upon which they can draw in order to communicate, and on the other hand how these are used in specific cases, in particular combinations, to communicate particular meanings. So in the case of Michael Jackson 'Billie Jean', rather than being satisfied with adjectives such as 'troubled', the aim is to describe the features of his music that allow us hear such things. On what repertoire of meaning potentials in term of melodic form, sound quality, vocal and instrumental articulation does Jackson draw upon to communicate meanings like 'tense' and 'obsession'? This social semiotic approach draws in the first place on the work of the linguist Halliday (1978) and Kress (2010) and has been successfully applied to other modes of communication, to images (Kress and Van Leeuwen, 1996), multimedia (Baldry and Thibault, 2006), three dimensional objects (O'Toole, 1994) and more broadly to sound (Van Leeuwen, 1999) and to popular music (Machin, 2010).

To draw out the underlying meaning potentials available to musicians this analysis draws extensively on the work of the musicologists Cooke (1959) who believed that it was possible to describe and inventorise the 'rule-book' of classical music. In other words he thought it possible to reveal the underlying principles of melody and rhythm upon which composers draw in order to communicate things like sadness, outpouring of emotion, entrapment, etc. Tagg (1983) has described the way that in our culture we have come to make associations of particular musical patterns and sounds with emotions, attitudes, settings and events through their repetition in our lives and as they have become embedded in our shared cultural conventions. A composer can therefore rely on a certain combination of notes as being heard by listeners as 'romantic' or 'scary'.

Tagg (1997) discusses the emergence of sounds and music as communicative acts in early human societies in terms of the way they could be used to express the attitudes and ideas associated with certain activities such as initiation rites, marriage ceremonies, harvests and the hunt. So analysis could compare the rhythmic intensity of the kinds of sounds used as members of a group prepare themselves for a hunt as when they wish to send a child to sleep. Tagg states:

Obviously, the pace required in conjunction with a hunt - intensity of heartbeat, speed of eye, of hands, arms, feet and breathing — will be far greater than that needed for singing a child to sleep (...) In the case of the hunt, quick, sudden movements enacted with the precision of split seconds are vital ingredients of the activity, but they would be detrimental when trying to send a child to sleep (1997:8) 
Just as Tagg seeks to identify what more specific elements are present in kinds of musical experience: quickness, suddenness, versus gentle and lingering, so the same kind of analysis can be carried out for popular music songs. But first we need to identify what exactly are some of the basic semiotic resources in music, in a social semiotic sense what are the basic building blocks available?

The analysis also draws specifically on the observation on the meaning of sound quality by Van Leeuwen (1999) who attempted to inventorize a list of speech qualities that comprise the available resources for speakers to create meanings not only through word choice but additionally through voice quality. His observations are combined here with the linguistic theory of Brazil, Coulthard and Johns (1980), McConnell- Ginet (1977) and musical theory of Schaffer (1977), and Tagg $(1982,1984,1994)$ in order to look also at the sound qualities of musical instruments. These analytical tools will be explained fully in each section.

\section{The semiotic meaning of sounds}

We have seen above that music is often described through adjectives. So we find words like 'tense' and 'obsessive' to describe the sounds of Thriller. The problem is that description and evaluation become mixed and the terms themselves are vague. They do not help us to identify and understand exactly what kinds of affordances the sounds are drawing upon. What exactly are the sound qualities that communicate 'tension' and 'obsession'? Drawing on Van Leeuwen (1999) and the cognitive psychologist Arnheim (1969) we can establish two kinds of origins for the meanings of sound qualities. These help to provide us with our first building blocks for identifying the affordances available to musicians which are then developed over the course of this paper.

\section{Provenance}

This is simply when a sound comes to have a particular meaning through cultural accumulation of associations. For example, to a Northern European listener pan pipes suggest 'nature' or simple, ancient cultures especially those from Latin America. The sitar is used to represent the whole of Indian culture or the esoteric and mysticism in general. Such associations may have no actual connection to time or place, for example, the bagpipes are associated with Scotland even though historians tell us they were only recently introduced early in the $20^{\text {th }}$ Century. So a pop artist might use a violin to connote folk and tradition or a synthesiser to connote technology and modernity.

The fact that we experience certain musical notes and note sequences as communicating specific emotions too lies in a cultural accumulation of associations. Tagg (1983) was interested in the way that certain music came to be able to represent different kinds of landscape and character in the $19^{\text {th }}$ century. These associations no longer appear anything but natural to listeners due to repetition. The music used in movies for romantic moments, drawing on this cultural history of sound, simply sounds 'romantic' to us when we hear it. However, while provenance explains why certain notes and note combinations have particular meanings it does not permit us to understand the meaning of how these notes are 
played, the qualities of these notes. It is the second of our categories that helps us to think about this.

\section{Experiential meaning potential}

The meaning of sound quality may also derive from associations of things in the real world. Arnheim (1969) argued that communication is steeped in 'experiential associations' (p117). He explains that 'human beings are naturally aware of the structural resemblance uniting physical and non physical objects' (p118). So we might clap our hands together to suggest a conflict of interest between two people. There is no actual clapping or physical collision going on in the interaction but communication works by drawing on an experiential association of these to understand something of the way that people may not agree. In the same way the sound associated with crashing objects could be thought to suggest conflict as opposed to a gentle drifting sound that might mean some kind of mutual attraction.

Our physical environment produces noises all the time, all of which have meaning to us. These may be due to certain qualities of the element that makes the sound or even its meaning to us in our lives. Thunder makes a booming sound which may mean violent weather or lightening, things that frighten us or present danger. The sound of thunder also gives the impression of vastness where the whole sky appears to be filled with the noise. This means that such booming sounds therefore when made artificially can be used to communicate something ominous, something powerful or massive. The opposite can be the case for softer, or higher pitched sounds. Tagg (1993) notes that humans tend to use sounds and sound qualities that allow us to draw on associations in the physical world to understand our social and personal experiences. So a smooth sound will be more likely to be used to represent something like romance that a very rough raspy one. The point is, as we will see, that this kind of observation gives us a starting point for looking in greater detail at the sound qualities of voices and musical instruments.

It also appears to be the case that much of the sound qualities in music along with our experience of musical rhythm itself may be linked to our use of language (Levitin, 2006). Our ears and brains are finely tuned not just to listen for the meanings of words and grammar but also to the manner in which these are delivered, to voice quality and to the rhythms in speech. In linguistics much research has documented this phenomenon (McConnell-Ginet, 1977, 1988; Bell and van Leeuwen, 1990). More will be said about both these kinds of origins of meaning in sound as we look closely at 'Billie Jean'.

\section{Social Semiotics of 'Billie Jean'}

In the rest of the paper we look at the meaning of voice quality, pitch, pitch ranges, note value and the sound quality of instruments. In each case the meaning potentials will be first drawn out and explained using a number of examples from other well know popular songs and then applied to 'Billie Jean'. It is through this process that we can establish the underlying meaning potentials, the affordances, available to musicians in order to understand Jackson's own specific choices. Also we should bear in mind that in music meaning is created through all of the features working together. For analytical purposes we will deal with each in turn, 
accumulating our understanding as we move through them. It is through this process that we will get access to the semiotic resources in 'Billie Jean' that communicate 'tension', 'obsession', 'troubled relationships' and 'emotional urgency'.

\section{Voice Quality on 'Billie Jean'}

Drawing on van Leeuwen's (1999) observations on voice quality that draws on a range of work in linguistics we can begin by describing some of the features of Jackson's vocal style on 'Billie Jean' although these will be built upon further throughout the paper. Van Leeuwen lists eight qualities of voice that account for basic sounds found in the sounds made by speakers and singers. Two of these are particularly relevant for 'Billie Jean'.

When we hear a vocalist such as Frank Sinatra, Sid Vicious, Bob Dylan, or Paul Simon it is not difficult for us to produce adjectives to describe the way they sound different. I asked a friend and they suggested that Sinatra has a more 'crooning', 'seductive' style. Vicious is 'aggressive' and 'in your face'. Dylan is 'wistful' and 'earthy' and Simon is 'gentle' and 'thoughtful'. The friend described Jackson's voice as 'excited', 'youthful' and 'innocent'. Van Leeuwen's inventory of voice qualities allow us to describe these differences using careful description as opposed to these vague adjectives that are often tied in with our perceptions of the artists themselves as much as description of sounds.

\section{Tension}

This simply describes the extent to which we speak or sing with an open or closed throat. When we become tense in everyday situations our throats tend to close up. This is what we hear when we say that someone's voice sounded tense. Of the singers mentioned above Sinatra and Simon tend to use open relaxed throats whereas Vicious and Dylan tend to use closed throats. Therefore a vocalist like Paul Simon can sound more thoughtful, gentle and relaxed. Dylan can sound troubled and Vicious can sound aggressive. On 'Billie Jean' we can hear that Jackson uses a lot of closed throat to communicate tension. These observations are also useful for thinking about degrees of tension and relaxation in the way that the instruments are played on 'Billie Jean'.

\section{Breathiness}

This is simply to do with the degree of intimacy suggested by a voice. To bring out the meaning potential here, we can think of the contexts in which we hear people's breath. This can be when they are out of breath and panting, because of some physical or emotional exertion or strain. It can also be in moments of intimacy and sensuality. When we hear a person's breath when they speak this may even be a moment of confidentiality as they whisper in our ear, or share their thoughts with us when they are experiencing emotional strain or euphoria. When we listen to the vocals of most rock singers we do not hear breathiness or the close texture of their voice. Intimacy is not what is important in such cases. We may however, hear the breath of Paul Simon on one of his more reflective recordings. On 'Billie Jean' Jackson often sings in short breathy gasps. This appears to communicate both intimacy and sensuality along with an element of euphoria. To some extent this may 
bring something more of openness about 'Billie Jean' not necessarily communicated in the lyrics themselves. At the level of sound quality there is evidence that is not simply an account of an obsessive fan but something in which Jackson is emotionally implicated and moved.

As regards musical instruments 'breathiness' is also a useful way to think about the way we can hear the sound of a drum striking clearly and sensually or hear gentle sounds of fingers moving across a guitar fretboard or the sound of strings ringing out. This can be contrasted to cases where instruments sounds merge in with the mix.

We will be applying these observations on Jackson's vocals on 'Billie Jean' shortly and later to instrumentation. But first we turn our attention to the way he uses pitch, pitch ranges and particular kinds of note combinations.

\section{The meaning of pitch}

Melody is comprised of changes in pitch over different ranges of notes. We begin by looking at the basic meaning of pitch, moving on to how different ranges and kinds of pitch movement can have meaning potential.

Pitch is simply how high or low a sound is. A scream would be a high note, thunder a low note. The meaning of pitch is rich in metaphorical associations. Cooke (1959) has suggested that high pitch means effort, low the opposite; in other words contained, immobile and static (p. 102). We could think of this metaphorically as being like someone speaking in a low deep voice as compared to raising their voice in excitement. But higher pitch can also extend to mean agitation and lower pitch mean low drooping despair. Cooke shows that classical composers have used high pitch to suggest "up and away" due to its energy and low pitch to suggest "closer, down and relaxation" (1959, p. 103). In Western culture we have the association of up meaning 'feeling good' and down meaning 'feeling down'. We transfer this meaning to pitch even though there is no reason why a high pitch should be thought of as 'high' at all as height is not involved. Cooke adds that pitches beyond the range of the human voice can, in the cases of higher tones, give a sense of the ethereal, lightness, transcendence.

We also have associations of the way things in our everyday world produce different pitches of sounds. Heavy objects can make deep sounds when they move or fall. Smaller animals might make higher pitched squeaking sounds. Deep sounds give a sense of danger or something ominous as in thunder. This could be why deep sounds are often used to symbolise gravity or danger. These all provide meaning potentials in terms of the way an artist might use a low pitch, like Tom Waits, or a high pitch, like Michael Jackson. One can seem closer to drooping despair, heavy with gravity or menace, immobile, while the other can appear much less robust, agitated and high energy.

More will be said specifically about this when we move on to the meaning of movement in pitch. In music, after all, pitches rarely stay at one level. We will also later be able to comment on the pitch used in instrumentation. 


\section{The meaning of ascending and descending in pitch}

We have listed a number of meaning potentials for high and low pitches. But often when we hear sounds and music there is generally movement in pitch. A vocal line will usually rise and fall in pitch. The direction and extent of this movement can also have meaning potential.

Cooke (1959) suggests that in classical music ascending melodies are associated with outward expressions of emotions whereas descending melodies are associated with incoming emotion. This is due to the association of higher pitches with higher levels of energy and brightness and lower pitches with associations of low levels of energy. The movement from one to the other expresses a shift in either direction. A movement from a high pitch to a low pitch the meaning is of a falling of energy. The opposite, a gradual slide from low to high pitch, gives a sense of a picking up of spirits. National anthems use stepped increases in pitch to suggest the brightness and energy of the national spirit. Anthems will often also use some lower pitches to suggest the weight and gravity of the nation.

We can show how increase and decrease in pitch works in a familiar popular song to help us to establish the available meaning potentials draw upon by Jackson on 'Billie Jean'. Here is the melody from the start of "Blueberry Hill". First I show the direction of the movement of notes under the lyrics and then represent this as a graph. On the graph the scale on the right represents the notes going up and down.

I found my thri-ill on Blue-ber-ry Hill



Blueberry Hill

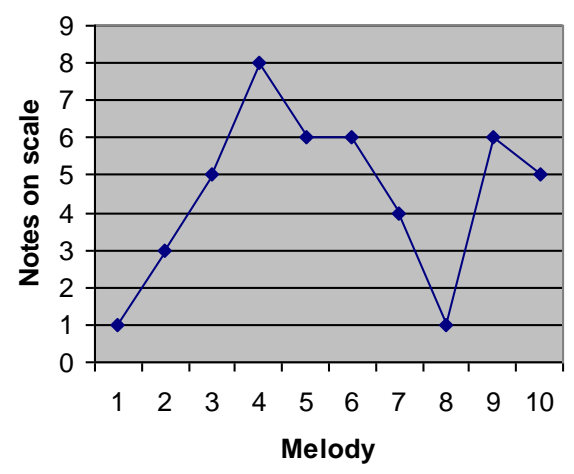

The arrows positioned under the lyrics indicate whether the melody of the song, the notes, are ascending in pitch, descending or staying at the same level. This can be seen clearly on the chart. In this case the first four words of the song ascend. If you hum the tune yourself you will be able to hear this. Therefore we can say that the singer here is expressing an outgoing emotion or an increase in optimism which is done with an open throat communicating no tension. The melody then levels off and descends back to its starting point, although there is an important ascending note before this on 'berry'. So the outward expression of emotion, of 
joy, is followed by a move back to something more grounded and relaxed, perhaps more thoughtful. But the other ascending note on the second syllable of 'berry' marks another burst of emotion before it tidily resolves back to its starting point, giving a sense of closure. If the melody descended from the start then, according to Cooke, there would be incoming emotion, such as a received sense of joy or consolation or even a simple slide to bleaker thoughts and self-absorption. So in terms of pitch 'Blueberry Hill' is an outburst of positive emotion.

While more needs to be said about pitch before we have a comprehensive set of tools with which to consider 'Billie Jean' we can look at the pitch movement in the first line of the song which is typical of the whole song.

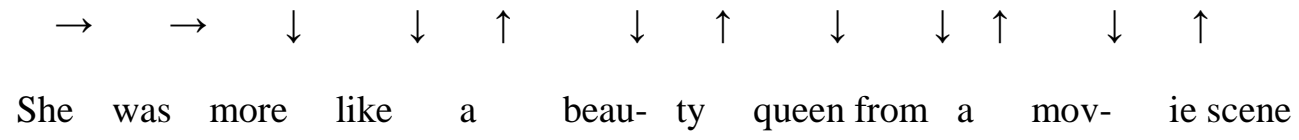

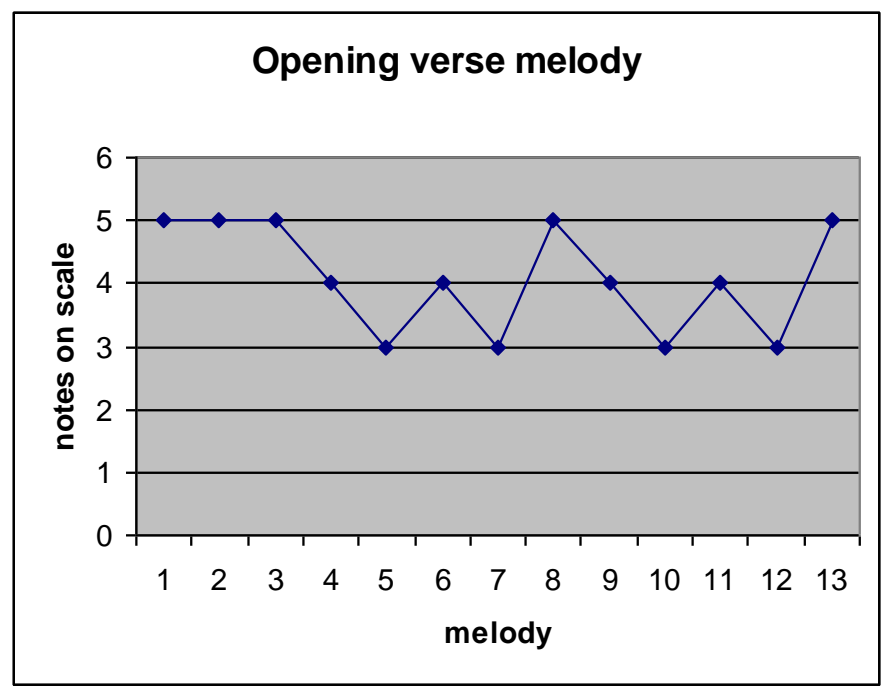

We can see that Jackson tends to not use longer consistent increases or decreases in pitch but jerkier pitch movements. There are therefore no clear outbursts of emotional energy nor falling off of energy. This gives a sense of emotional instability, and since there is often tension in his throat as he sings these words along with breathy gasps we get a sense of a mixture of agitation and as we will see, also a kind of euphoria. The meaning of this can be drawn out more when we consider next the quality which is pitch range.

\section{What Is the Range in Pitch Between the Highest and Lowest Note?}

As well as whether pitch increases or decreases there is important meaning potential in the range of these changes. A large pitch range means letting more energy out whereas a small pitch range means holding more energy in. So we might think of a singer like Bob Dylan holding energy in, while a singer like Freddie Mercury lets it out as on songs like 'Find me Somebody to Love'. Linguists Brazil, Coulthard, \& Johns (1980) argue that wider pitch range in speech is akin to excitement, surprise, and anger. Narrow pitch range associated with 
boredom and misery. Pitch range in speech is also associated with emotional expressiveness. In Anglo-American societies, men have less pitch range than women (McConnell-Ginet, 1977, 1988). So small pitch ranges can be associated with holding in, or even modesty. In Bob Dylan's singing about tragedy or injustice, using a limited pitch can give a sense of resignation or contained pain, perhaps giving it a different kind of gravity than an opera singer using a large range to sing about the same subjects. Before we can think about how this applies to 'Billie Jean' we need first to look at how we can examine pitch range. We can most easily show this if we return to our example of 'Blueberry Hill'. Here we look at the way the melody uses notes from a scale of 8 notes in order to show pitch range.

Songs normally draw on a scale which is comprised of 8 notes. Note 1 gives the name of the scale and is called the root note and the 8 notes after this rise in pitch. Since there are 8 notes in the scale, note 8 is the same as the root but an octave higher. We can see this on the illustration of the piano key board below.

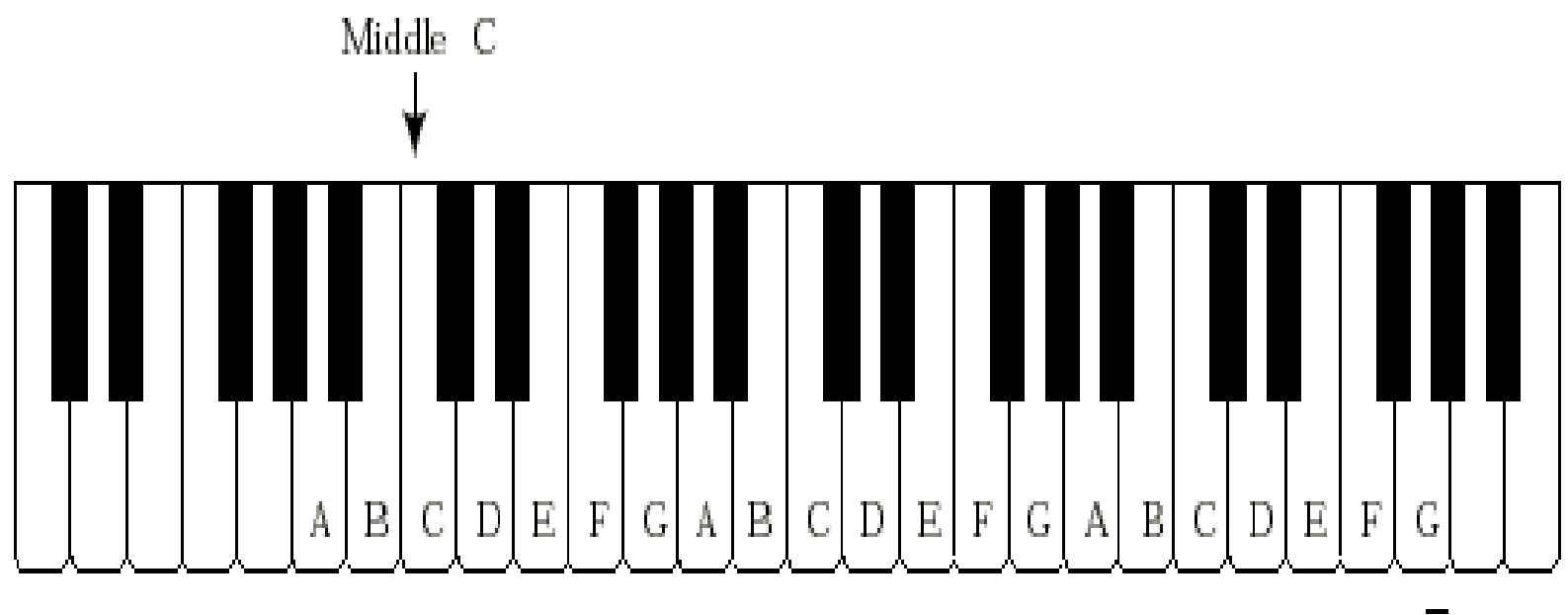

Fig. 1: 8 notes rising in pitch on piano keyboard

Middle $\mathrm{C}$ is note 1 . If we move up 8 notes to the right we again come to $\mathrm{C}$. If we move 8 notes down 8 notes to the left we find the same sequence of notes repeated.

Below the notes in 'Blueberry Hill' are represented with numbers to indicate where they are in the 8 notes of the scale. In this case the higher the number, the higher the pitch of the note, although if we see a 1 followed by a down ward arrow and then a 7 this would mean that the melody had descended to the $7^{\text {th }}$ note below.

I found my thr- ill on Blue-ber---ry Hill

\section{$1 \uparrow \begin{array}{llllllllllllll} & \uparrow & \uparrow & 8 & \downarrow & 6 \rightarrow 6 \downarrow & 4 \downarrow & \uparrow & 6 \downarrow 5\end{array}$}

What we find in 'Blueberry Hill' is a large pitch raise in the first phrase 'I found my thrill'. Here there is a leap of 8 notes. This is therefore an extensive release of energy and brightness. Following Cooke (1959) we can say it is large outward expression of energy. This interpretation suits the lyrics, which seem to be highly positive. However, if the notes 
had descended or been over a limited range, it would have resulted in a very different expression of those lyrics. So here the melodic form communicates a celebration of the event of finding the thrill; a descending pitch would perhaps lend a mourning, pining quality to the song a smaller pitch range something more emotionally restricted.

Below is an example of a song that uses a descending melody. In "Babylon" by David Gray we have an example where each line of the verse descends. Here is one of the lines:

Fri--day night and I'm go-ing no where

$3 \rightarrow 3 \rightarrow 3 \downarrow 2 \downarrow 2 \downarrow 1 \rightarrow 1 \rightarrow 1 \downarrow 6$

Babylon verse

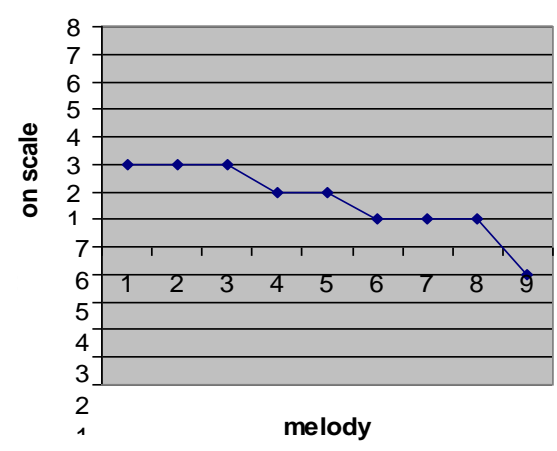

Here the melody begins on the $3^{\text {rd }}$ note and then descends in steps as can be seen on the chart. This suggests the opposite of outgoing emotion. This can give a sense of not particularly attempting to reach out to communicate as well as a falling of mood. We can imagine that if the same lyrics were sung with an ascending melody it would seem as if he were pleased he was going nowhere.

Some melodies neither ascend nor descend but are very static. One example of this would be 'Anarchy in the UK' by the Sex Pistols. Much of this melody takes place with repetition of the first note. There is therefore very little outward giving of emotion or positive energy. Nor is there any falling away of energy. This means that there is something very contained, restricted or confident about the way it is sung. In fact the vocalist sings the song generally at a high pitch which conveys an emotional intensity. Yet in this intensity there is no emotional outpouring or pleasure as in "Blueberry Hill". There are only short sharp occasional outbursts to the fourth note towards the end of each line. .

For 'Billie Jean' we find much stasis in the melody with repeated notes and smaller pitch movements. There is a combination of emotional containment or entrapment and jerky melodic movements. We can see this in the case of the first verse: 
She was more like a beau- ty queen from a mov- ie scene

$5 \rightarrow 5 \rightarrow 5 \downarrow 4 \downarrow 3 \mathrm{~m} \uparrow 4 \downarrow 3 \mathrm{~m} \uparrow 5 \downarrow 4 \downarrow 3 \mathrm{~m} \uparrow 4 \quad \downarrow \quad 3 \mathrm{~m} \quad 5 \rightarrow$

I said don't mind but what do you mean I am the one

$5 \rightarrow 5 \rightarrow 5 \rightarrow 5 \downarrow 4 \downarrow 3 \mathrm{~m} \uparrow 4 \downarrow 3 \mathrm{~m} \uparrow 5 \downarrow 4 \downarrow 3 \mathrm{~m} \downarrow 2 \downarrow 1$

We have already seen the graph for the $1^{\text {st }}$ line above. Here is the graph for the second line:

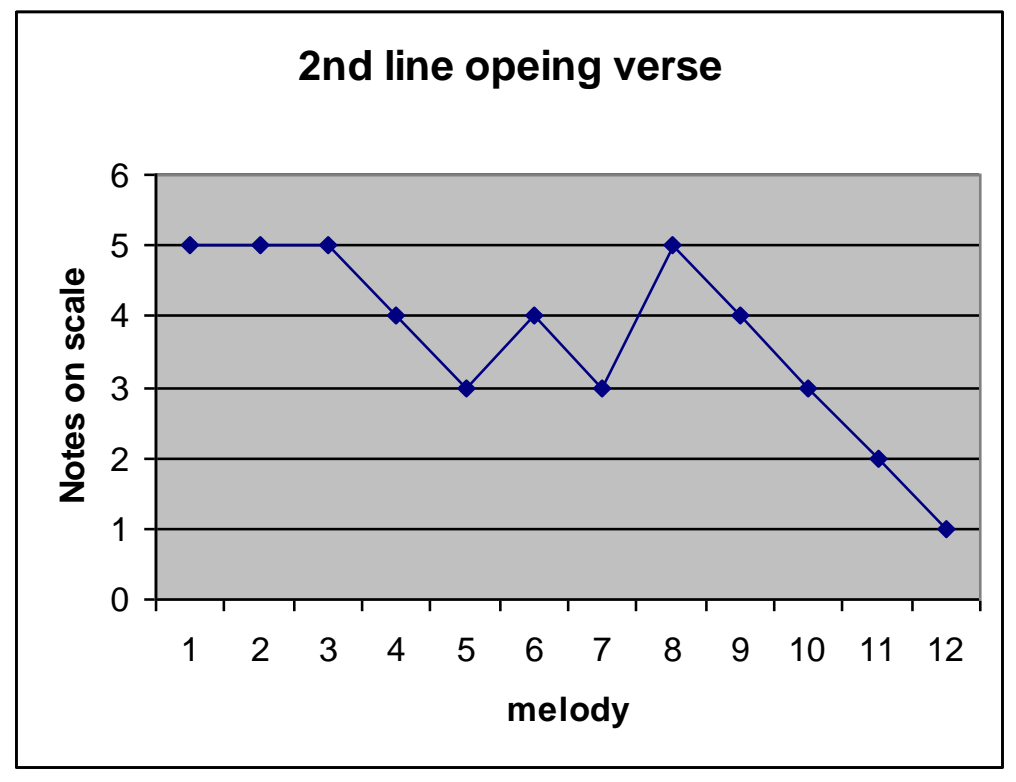

Much of this verse is characterised by jumpy melodies, with sections of stasis. It is only on the words 'mean, I am the one' where there is a clear descending pattern. Overall these jerky melodies sung at a high pitch give a sense of excitement and high energy, of lightness, and perhaps fragility, yet without clear direction. The jerkiness brings a sense of uneasiness of not staying still. This is also communicated through the sharp breaths and tension in the voice. We can see the same on the lines which follow the verse which start "who will dance...'

Who will dance on the floor in the round

$1 \uparrow 2 \downarrow 1 \rightarrow 1 \uparrow 2 \downarrow 1 \rightarrow 1 \uparrow 2 \downarrow 1$ 


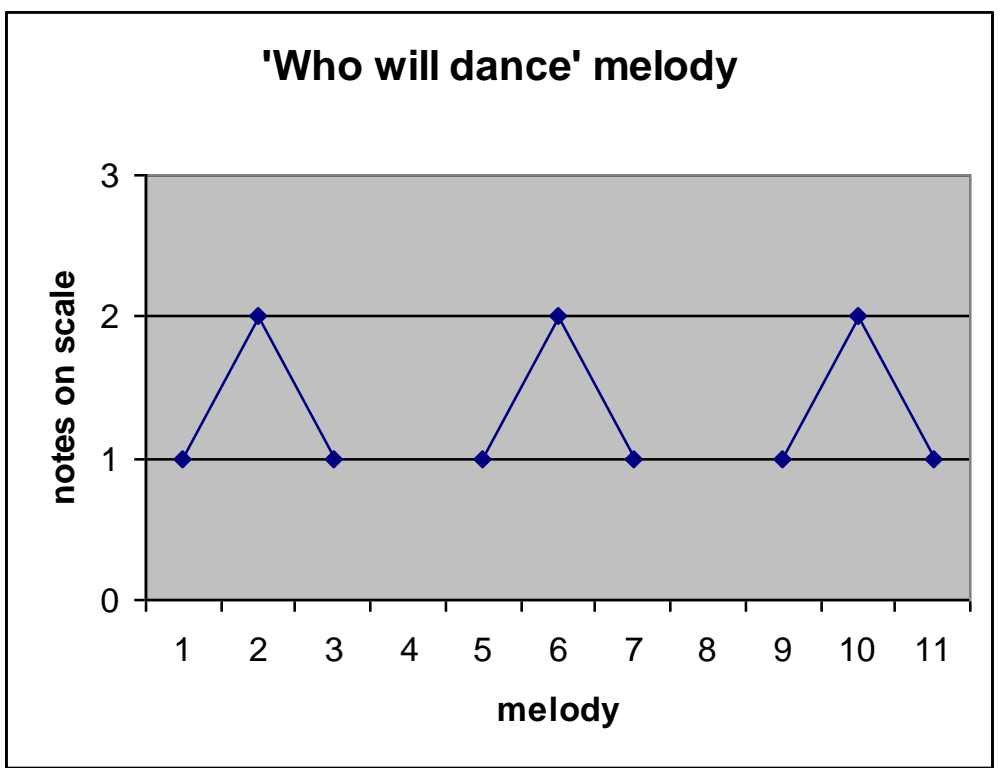

These are sung as bursts of energy, but with no emotional expansion. This use of short bursts in what we can call 'disjunctive articulation' has important meaning potential. Some melodies will be sung in a way such that words are joined together in longer phrases as we find for 'Blueberry Hill' where there is less a sense of an exclamation as an emotional lingering. There are long extended rises and falls in pitch, often with extended gentle decreases in pitch over a word. 'Billie Jean' is different, and it is here that we get a further hint at the tension and obsession in the sounds along with the more fragile and almost anxious higher pitches.

Bell and Van Leeuwen (1993) have noted that these kinds of shorter phrases are associated linguistically with sincerity, certainly, weight and therefore with authority. We can imagine the effect if someone were to speak to us in sentences comprised of bursts words. This is common to the way that news readers speak, for example. In the case of news readers it can also connote the urgency and immediacy of news. We might therefore be less surprised to hear folk singers using such short bursts to communicate sincerity. The opposite case where singers produce longer lingering statements suggests rather slow burning internal emotion.

In the case of 'Billie Jean' we find that Jackson uses these shorter breathy bursts but combines them with high pitches and tension. We can imagine the effect if a news reader combined the shorter phrases with such higher pitches. Simply this would connote a level of sincerity and weight, but at a level of emotional involvement and fragility inappropriate for news reading.

\section{The meaning of using different notes of the scale}

This is the part that the non-musician may find slightly more off-putting. But it is very simple and technical terms are avoided. As pointed out above there are eight notes in a scale 
and each, as Cooke (1959) has shown, has its own meaning potential. Jackson's note choices for 'Billie Jean' are one important way that 'trouble' and 'harrowing' are put into the music.

A pop song is normally based on one scale in which there are 8 notes. So in a scale of $\mathrm{C}$ there are eight notes following from note one, $\mathrm{C}$. The eighth note, the octave, marks a return to $\mathrm{C}$. In any song both the notes in the melody and the notes in the chords of the accompaniment are drawn from these notes. In between these eight notes are other notes that do not belong to the scale, but to other scales. These can be used in songs to add more drama and trouble. For none musicians it is worth pointing out that this division of notes in this way, into eight notes with specific identities, is completely arbitrary and can be explained by certain cultural and historical developments. Nevertheless due to repetition these now sound natural and inevitable to our ears (Tagg, 1983).

To make up a melody any of the eight notes can be used. But each of the eight notes has a different kind of sound which in turn has a different kind of effect for the listener. Because they have different effects, certain notes and certain note combinations are usually used. Tagg suggests that since we have been hearing these combinations and making certain associations for a long period in our culture, we can now easily recognise what is being communicated, what mood, what idea, what emotion.

In melodies certain notes of the eight in any scale are used a lot as they create a solid connection to the musical accompaniment, which will also draw on the same notes. These commonly used notes are mainly notes 1 and 5 . Note 1 is the main defining note of the scale. In the scale of $\mathrm{C}$ therefore, note 1 is $\mathrm{C}$. So this anchors the melody to the scale firmly and roundly.

Note 5 is similar in sound to note 1 and therefore is also good for anchoring the melody to the scale. Also important is note 3 . These structures using notes 1, 3 and 5, have become the basis of western music.

Notes that anchor the melody to the scale and to the accompaniment allow the music to feel 'easy' or 'rounded'. In contrast jazz will use many notes that do not create this solid connection in order to create tension. Blues music often uses the occasional difficult, or "blue," note to create and release tension. I have heard music of boy bands which have at no point deviated from the $1^{\text {st }}, 3^{\text {rd }}$, such is the importance of creating an 'easy' sound.

If we look at the melody of 'Blueberry Hill' above we can now think about exactly which notes are used in the melody. The first four notes are $1,3,5,8$. Since the $8^{\text {th }}$ note is the same as note 1 we can say the first four notes are $1,3,51$. Such a combination will anchor the melody, the song, to the music underneath very roundly. There is no complexity, trouble or doubt in what the vocalist expresses. In the case of 'Billie Jean' we can see on the opening verse that there is also the extensive use of the $5^{\text {th }}$ and $3^{\text {rd }}$ notes. Throughout the song these are important along with the $1^{\text {st }}$ note. At this level we might say therefore that the song is highly grounded. 
Interestingly the verse of 'Billie Jean' begins not on the $1^{\text {st }}$ note as do many popular songs but on the $5^{\text {th }}$, only resolving to the $1^{\text {st }}$ note at the end of the line on the word 'one'. This is interesting for two reasons. First, using the $5^{\text {th }}$ in this way is a technique often used in jazz. An instrument will play a $5^{\text {th }}$ in place of a $1^{\text {st }}$ to give a sense of space, distance or very slight tension in the music. So Jackson begins telling the story with this kind of mood, of slight tension and distance. Second, it is notable that the $1^{\text {st }}$ note, the grounding note, is first used in the melody only when Jackson sings the words 'I am the one'. This seems to make him only truly grounded at this point through being the 'one'. And it is here that he sings out the words with much less jerkiness, less breathiness and with an open throat. In Cooke's model this would suggest he may therefore have an ambiguous relationship with this situation. At first listening the lyrics appear to be a warning about fanatical fans. But melodically there is a suggestion that it may be through this process of being the 'one', the target of the obsession, which he truly finds himself, where he may have less gasping, nervous tension. As we have already seen the melody appears to indicate high levels not just of sincerity but also of instability and excitement. It seems that it is not just a warning story but also a view into Jackson's subjectivity.

The $3^{\text {rd }}$ note is important for other reasons. It is the way that the $3 \mathrm{rd}$ and other notes are used that can bring much more complexity of emotion and feel to a melody. You might be aware that, in general, a minor key is sad and a major key is happy. Research suggests that major notes and chords are associated with positive feelings. A minor key is created by lowering some of the notes in the eight-note scale; the 3rd and the 7th notes. These are lowered to notes in between the notes of the scale. So they become a bit like a 2 and a half and a 6 and a half. If a melody has the standard 3rd and 7th notes then it is a major melody and is therefore happy and joyful. If it has the lowered 3rd and perhaps a lowered 7 th, it is sad.

Looking at the example of 'Blueberry Hill' above we can see that the 3rd note used for 'found' is a standard 3rd, also known as a major note. The song goes straight to this defining happy note from the 1st note. Therefore it is happy. Since it is an ascending melody we can comfortably say that we have an outward expression of joy and brightness. In contrast had this note been a minor $3^{\text {rd }}$ the word 'found' would have sounded very sad and regretful. If you listen to the opening line of 'Ain't No Sunshine', you can here this kind of sadness. This song opens, like 'Blueberry Hill' with an ascending melody but uses the minor rather than major $3^{\text {rd }}$. The pain of this song therefore lies in the melody as well as the lyrics. Of course there is nothing 'painful' about the minor $3^{\text {rd }}$ in itself. It is just an association that has become established through semiotic provenance.

The 4th is a note that Cooke suggests is associated with building or moving forwards. It can also give a sense therefore of space and possibility. In the case of the Sex Pistol's 'Anarchy in the UK' the verse melody is comprised of the $1^{\text {st }}$ note which repeats for most of the verse and the $4^{\text {th }}$ note. So while the melody is dominated by stasis, which we suggested in this case could mean confidence or at least contented lack of interest, there is a repeated $4^{\text {th }}$ to suggest some building. The meaning would have been very different had the first note always moved towards a minor $3^{\text {rd }}$ or minor $7^{\text {th }}$. 
The $2^{\text {nd }}$ note has been associated with transition by the suggestion of movement, or the promise of something to follow, or lengthened $2^{\text {nd }}$ notes can suggest limbo or entrapment. This is because of its position between the strongly related $1^{\text {st }}$ and $3^{\text {rd }}$. 'Aint No Sunshine' uses a $2^{\text {nd }}$ note for the word 'when' for the words 'when she's gone'. The lingering on this note suggests a sense of limbo.

The sixth note is very much like the major $3^{\text {rd }}$ and can therefore be used to ground the melody. On 'Blueberry Hill' we find much use of the $1^{\text {st }}, 3^{\text {rd }}$ and $5^{\text {th }}$ which give the melody a grounded solid feel and also the $6^{\text {th }}$ which has the same effect. The only note that does not have this grounding effect in this melody is the $4^{\text {th }}$ used for the word 'Blue' which suggests building.

If we look at the verse and chorus melodies on 'Billie Jean' we find an extensive use of sad and painful minor notes along with $4^{\text {th }}$ notes. We can begin with the opening lines which were illustrated above.

The opening vocal line is a confined melody mainly based around the limited pitch range of the 5, 4 and $3 \mathrm{~m}$. There is a combination of stasis through repetition of the same notes several times and of shorter fluctuations in melody which, in contrasts to songs like 'Bluebery Hill' does not suggest steady outpouring or receiving of emotion. This fluctuation is characterised both in the melody and in the high pitched disjunctive articulation of how they are delivered by Jackson in tense, breathy bursts rather than more legato, smoother articulation as we find on 'Blueberry Hill. What is communicated throughout the 'Billie Jean' melody is emotional instability with some stasis, communicated with a level of sincerity, tension and high excitement.

The opening verse is anchored through the $5^{\text {th }}$ note but since this is used from the start this brings a slightly floating effect. The $1^{\text {st }}$ note is used only at the end of the line for the word 'one'. So it is only here where it is truly grounded and resolved. It first passes through the $3 \mathrm{~m}$ note on the word 'am' indicating some painful about this fact. We find an extensive use of the building $4^{\text {th }}$ note in the melody. In combination with the extensive use of $3 \mathrm{~m}$ notes this creates a building sense of sadness and pain. And this raise to the painful note along with the high pitch and tense throat gives a sense of euphoria. While this is painful Jackson appears to be highly stimulated by this fact.

The linking section 'Who will dance, on the floor, in the round is comprised of small bursts of breathy, sensual energy using the $1^{\text {st }}$ and $2^{\text {nd }}$ note. This again creates a jerky, nervous effect. The $2^{\text {nd }}$ note is appropriately used for the asking of a question.

For the next section which begins 'People always told me' we find an increase in pitch of a whole 8 notes. This suggests increased energy. 
$7 \mathrm{~m} \uparrow 1 \downarrow 7 \mathrm{~m} \uparrow \downarrow 7 \mathrm{~m} \uparrow 1 \rightarrow 1 \rightarrow 1 \uparrow \quad 3 \mathrm{~m} \downarrow 1 \rightarrow 1 \rightarrow 1 \downarrow 7 \mathrm{~m} \uparrow 1 \downarrow$
And mo-ther $\quad$ al-ways told me be $\quad$ care-ful of who you love.

$5 \uparrow 1 \downarrow 6 \rightarrow 6 \rightarrow 6 \rightarrow 6 \uparrow 7 \mathrm{~m} \quad 1 \rightarrow 1 \rightarrow 1 \downarrow 7 \rightarrow 7 \rightarrow 7 \uparrow 1 \uparrow 2$

And be careful of what you do 'cos the lie be comes the truth

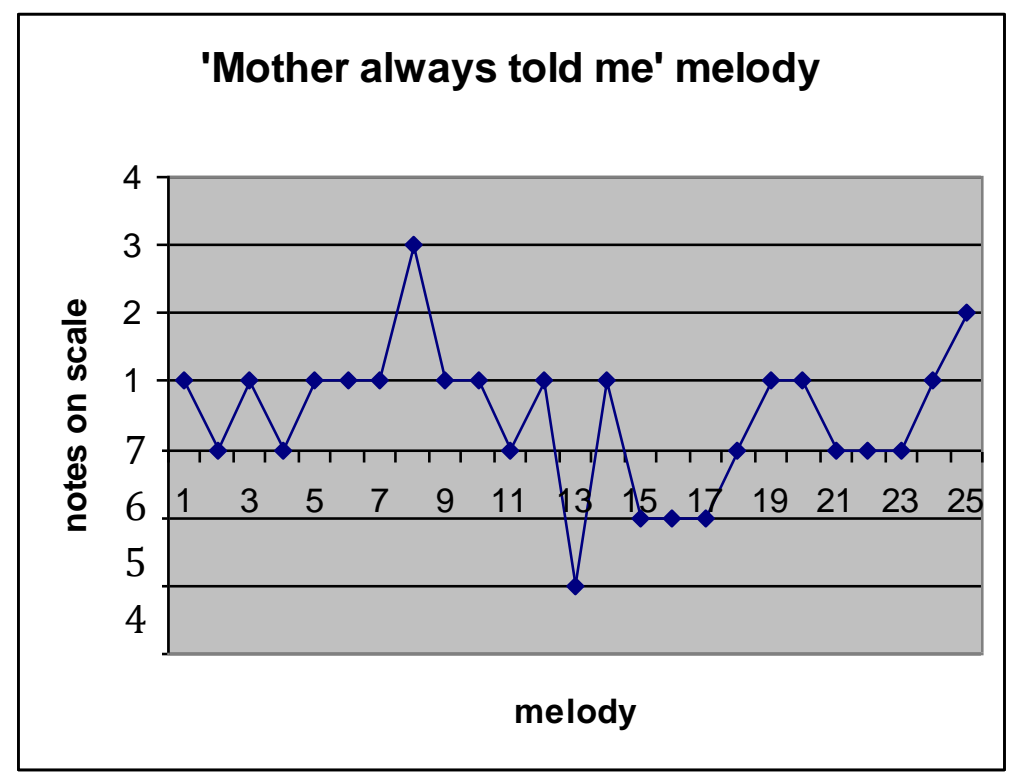

The first section is comprised mainly of stasis with movement between the $1^{\text {st }}$ note and the painful $7 \mathrm{~m}$. There is one burst of energy leaping to the sad $3 \mathrm{~m}$ note on the word 'Careful' and then a return to the painful stasis. The second section has much more emotional range. It starts by falling down to a grounding $5^{\text {th }}$ note which brings a sense of gravity for 'And be careful' and then stays on the relatively positive $6^{\text {th }}$ note, suggesting this is good advice. It then returns to more stasis over the $1^{\text {st }}$ and the $7^{\text {th }}$ notes again but this time interestingly not using the $7^{\text {th }}$ minor but the natural $7^{\text {th }}$ note which jars a little alongside the minor key. So the words 'lie becomes the truth' sound dischordant and uncomfortable. The line ends on the $2^{\text {nd }}$ note which gives a sense of something to follow, as if this was the prophecy that was fulfilled.

Overall here we have a surge in pitch and energy levels yet a continued use of stasis and emotional confinement and more jittery, nervous melodic pitch changes. There is a dominance of painful minor notes and the uncomfortable $7^{\text {th }}$ used to express the nature of lies becoming truth. In the lyrics too it is unclear of what has actually taken place and who has seduced who.

For the chorus we find less erratic melodic movement. 
$1 \rightarrow 1 \downarrow 7 \mathrm{~m} \downarrow 5 \uparrow \quad 1 \rightarrow 1 \rightarrow 1 \downarrow \quad 7 \mathrm{~m} \downarrow 5$

Bi-llie Je- an is not my lov- er

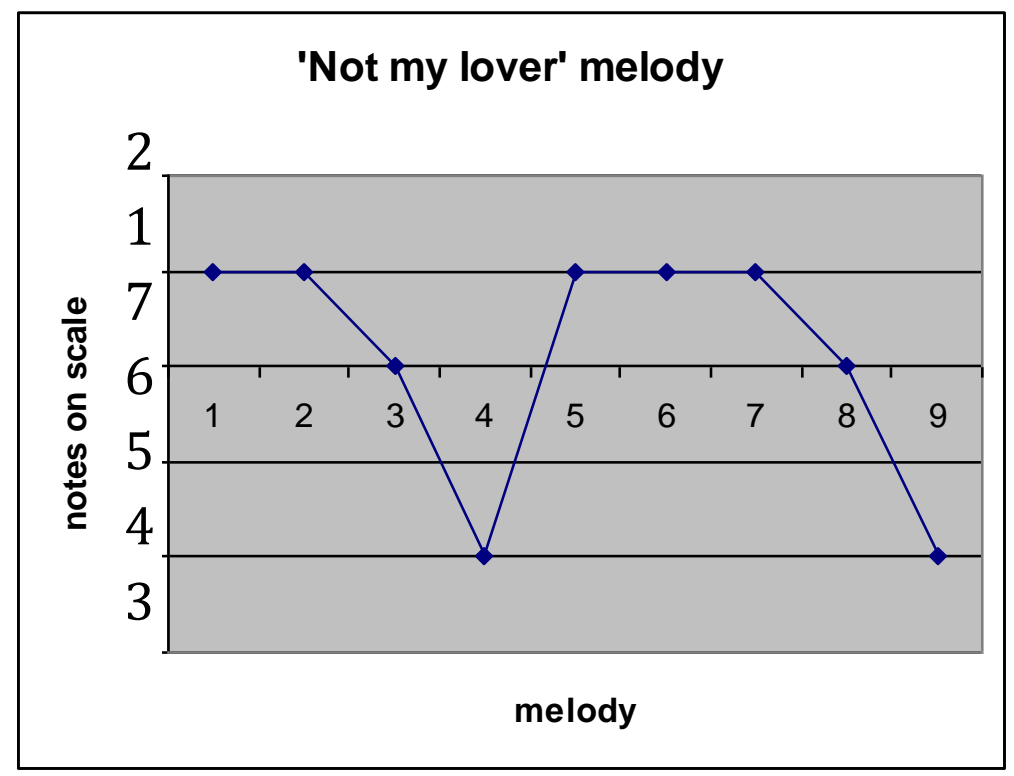

$1 \rightarrow \quad 1 \rightarrow 1 \downarrow 7 \mathrm{~m} \downarrow 5 \rightarrow 5 \uparrow \quad 1 \uparrow 3 \mathrm{~m} \uparrow 4 \downarrow 3 \mathrm{~m} \downarrow 2 \downarrow 1$

She's just a gi- $r l$ who claims that $I$ am the one

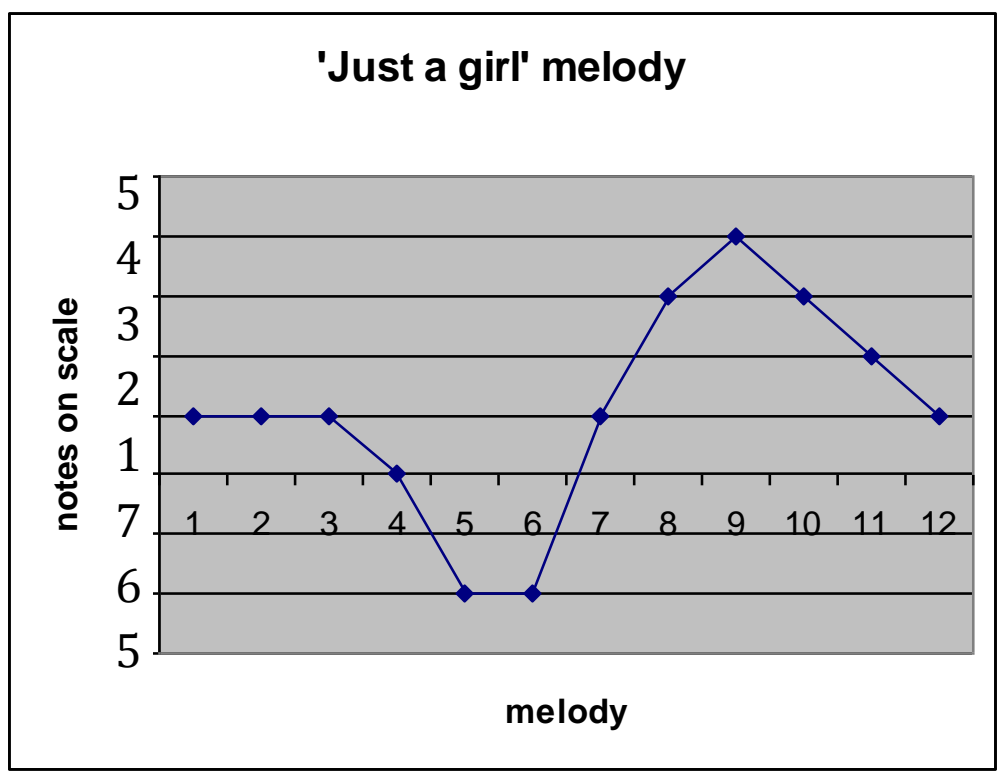

The chorus line starts with a falling away of pitch sinking straight to the painful $7 \mathrm{~m}$ note and then to the $5^{\text {th }}$ which repeats. This suggests a falling away of energy, a feeling of regret. Here the emotions are much more identifiable that for the verse and there is smooth legato articulation rather than the nervous bursts of phrasing for the verse. But what it interesting is 
the large surge in pitch for the lines 'claims that I am the one'. This uses a $3 \mathrm{~m}$ which makes it sad and also the $4^{\text {th }}$ note which suggests something building and possible ominous, but also communicates a sensed of building excitement again with the high pitch. But this then resolves back to the $1^{\text {st }}$ for 'I am the one'

We then have the following lines that repeat throughout the song:

$1 \uparrow \quad 3 \mathrm{~m} \uparrow 4 \downarrow 3 \mathrm{~m} \downarrow 2 \downarrow 1$

She said I am the one

This line goes straight form the $1^{\text {st }}$ note to the $3 \mathrm{~m}$ which for Cooke would suggest heavy outward expression of pain. It then goes to the building $4^{\text {th }}$ and descends back through to the $1^{\text {st }}$ note. Since the resolution to the $1^{\text {st }}$ note goes through the $3 \mathrm{~m}$ from the building $4^{\text {th }}$ we can see the menace in this accepted state of affairs suggested by the resolution. Here it is the $4^{\text {th }} 3 \mathrm{~m} 21$ combination that bring the beautiful sound.

$1 \rightarrow 1 \uparrow 5 \downarrow 4 \downarrow 1 \downarrow 6 \downarrow 5$

But the kid is not my son

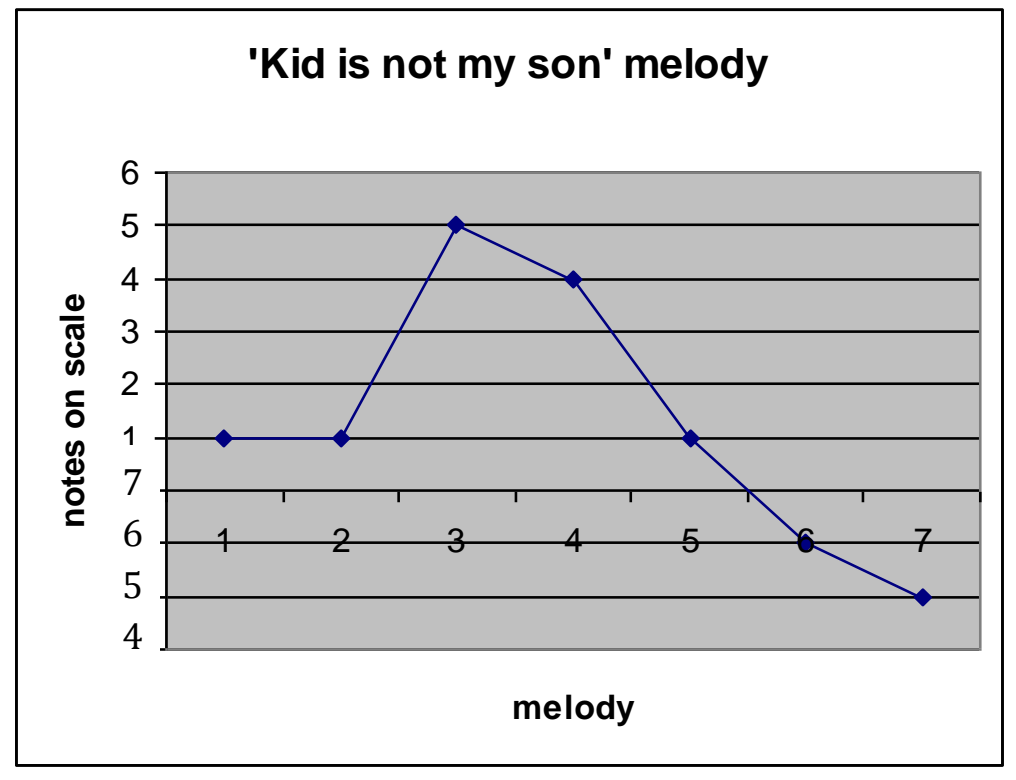

I find this a particularly beautiful section. Here we have a much more grounded melody, the most 'easy' and 'unproblematic' of the whole song with the use of the $1^{\text {st }}$ and $5^{\text {th }}$ notes and the $6^{\text {th }}$ note which sounds very much like the happy major $3^{\text {rd }}$ note. And we find the resolution here dipping lower in pitch than the $1^{\text {st }}$ note as if to give weight to what he says or possibly simply a sense of resolution. There is also the use of the $4^{\text {th }}$ which here suggests moving forwards. And importantly on the chorus we have less breathiness and more openness to the throat in the vocals. The chorus therefore shifts away from the higher levels of nervousness expressed in other sections of the song. 


\section{Pitch and sound quality on instrumentation}

Overall in terms of vocal melody and voice quality we find mainly nervous agitation and euphoria with limited pitch ranges suggesting emotional confinement but at the same time lack of stability within this. What is important to now consider is the way that this vocal line fits with the instrumental sounds. In this section we make a number of observations on the bass line and the synthesizer stabs using the observations on melody and sound quality developed over the preceding sections.

\section{Bass Line}

The bass line, to use adjectives in the first place, is a riff that cycles and gives a sense of relentlessness. Here is the melody which is repeated, cycling, throughout most of the song. It appears after a short introduction where we hear a synthetic dance drum beat:

$1 \downarrow 5 \uparrow 7 \mathrm{~m} \uparrow 1 \downarrow 7 \mathrm{~m} \downarrow 5 \downarrow 4 \uparrow 5$

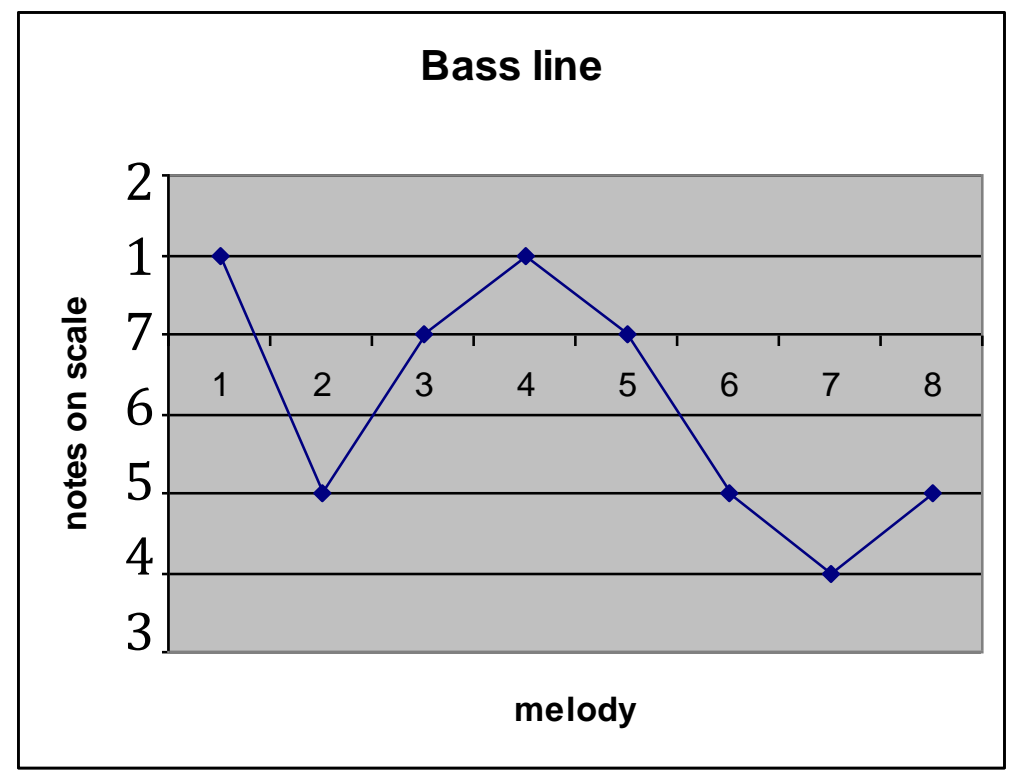

This is a grounded melody using lots of 1 and 5 notes. That the melody takes place in pitches lower than the starting $1^{\text {st }}$ note brings a sense of gravity through the metaphorical meaning of lower pitches. For mood it uses no 3rds but uses the painful $7 \mathrm{~m}$. There is also the use of the $4^{\text {th }}$ note at the lowest point in pitch to create a sense of building menace. The pitch range is not extensive and takes place over 4 notes. It is not therefore particularly emotionally expansive. The actual sound quality of the bass notes are relatively softly played and do not ring out. This relentlessly repeating cyclic bass riff with the lower $4^{\text {th }}$ note appears to give a creeping but relentless movement to the song, always moving forward and never staying still on one note and which remains just in the background pushing the song forwards. It is over this forwards movement that the vocal melody places its relative stasis and emotional instability at high levels of excitement and energy. It is as if Jackson is carried along by this momentum, gasping and agitated. 
Both Tagg (2001) and Schaffer (1977) have discussed the relationship between melodies and backing instruments and argued that certainly in popular music lead vocals or instruments either merge with or rise above the sounds and rhythms of the backing as a representation of the individual's place in society. In rock music guitars and drums are played at high volume and then the singer screams out above them when with amplificiation technology this is in fact unnecessary. The authors suggest this reflects a sense of the individual's place in the anonymous post industrial society where we need to shout to be heard, although even so our voices are only just heard. In this sense we might say that this bass line of 'Billie Jean' represents the social context in which Jackson finds himself - one that does not drown him out as is suggested by the backing of rock music, but one that moves him along relentlessly and with slightly darker undertones, but nevertheless with a stylish energy. .

\section{Synthesiser stabs}

The synthesiser stabs are played once the momentum of the bass line is established. These stabs are basically three chords that repeat returning to chord one. Chords are basically notes taken from the scale, usually the 1,3,5 and 7. These can be played in any order of pitch. The way the chords are played on 'Billie Jean' allows for a melodic sound to come from the sequence where the highest notes sound. This makes for a melody of notes $1,2,3 \mathrm{~m}, 2$. It is very compact and limited in pitch range suggesting a kind of emotional confinement. As with the bass line there is a sense of repetition here, of entrapment or of relentlessness.

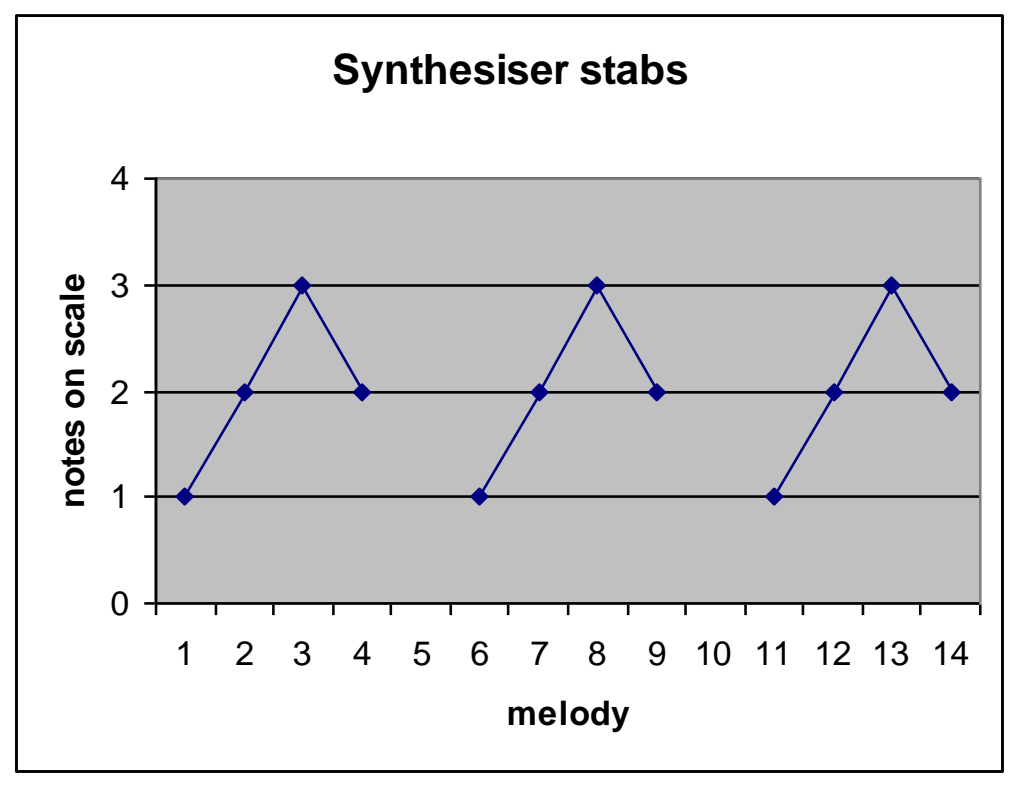

Melody notes on synthesiser stabs

What is of great importance is the way that these notes are played. The synthesiser chords are not played in a way that allows the notes to ring out which may have suggested an emotional release. They are played with a degree of tension that suggests hesitation. The metaphorical meaning potential here relates to tension versus relaxation in voice quality as discussed above. These stabs over a very confined pitch range are placed against the relentless bass line. The 
use of the $3 \mathrm{~m}$ note provides them with a degree of pain. So there is a painful hesitation or warning. In the model provided by Schaffer (1977) we might argue that this represents another layer in the social world occupied by Jackson. There is the relentlessness and menace of the bass line and the hesitant slightly tense, painful emotionally confined synthesiser stabs. Does this mean that Jackson finds himself in a world almost out of his control, where he is a passenger? Here the synthesiser stabs might communicate the soft yet creeping nature of the threats that face him or even himself attempting to negotiate them. It is over these that he places the melody and sound qualities of the vocals used to tell the story. It is over this relentless bass line and hesitant confined synthesiser that he places his nervous, energetic, sincere and sensuous story.

In sum the lyrics of 'Billie Jean' tell of an obsessed fan who appears to claim that Jackson is the father of her child which he says calmly is not the case. He is warned by people and his mother to take care of such situations, with some rather sad moments where his mother tells him to be careful of who he loves and lies can become truth, yet it appears he has been drawn in to some extent and it is unclear to what extent he is an agent in the process. The instruments provide a relentless grounded yet slightly menacing bass pulse along with a hesitant tense synthesiser over which he gasps and shifts around agitated and breathy in small jerky pitch shifts with lots of painful notes and also building notes. The story is told at higher pitches through tense throat communicating high energy, agitation, excitement and anxiety and at other times a breathy intimacy and sensuality or even arousal. Only in the chorus do we find more calm articulation and clear emotional direction. Moments of high energy and grounding appear to take placed when he tells us that he is 'the one'. Through these combinations we certainly gain a sense of 'tension', of 'obsession' of 'trouble' and of 'emotional urgency'.

\section{Conclusion}

Unlike visual and textual stimuli sound is something which enters our bodies. We can literally resonate with a thumping bass line or riff of an electric guitar. Sound can pass into and through our bodies and become part of us. We don't simply observe it at a distance as we can a picture on a screen or words on a page. In this way sound has a more immersive quality than other kinds of perception, than other acts of human communication. Sound and music can feel like they are a part of us, inside us. In this sense sound and music form a very special kind of set of semiotic resources that have this power to literally enter us and move us. In this paper I have attempted to take a step in exploring the way that Michael Jackson uses sound alongside lyrics on 'Billie Jean'. If we wish to understand the way that musicians communicate subjectivities then we need especially to understand how they communicate through sound as while through lyrics they can certainly communicate ideas these enter our bodies on a different level allowing us to resonate with them. If we wish to understand why we are so moved by certain musicians we should think more closely about the exact semiotic choices they make. We like to understand he linguistic and stylistic patters in literature which can mean analysing grammar and word choice. In the same way a semiotic analysis of music can help us to appreciate how musicians use the semiotic resources in sound in the same kind of special, creative way. 


\section{References}

Arnheim, R. (1969) Visual Thinking, CA: University of California Press.

Baldry, A. and Thibault, P.J. (2006) Multimodal Transcription and Text Analysis, London, Equinox

Bell, P. and Van Leeuwen, T. (1993) The Media Interview, Kensington, University New South Wales Press

Brazil, D., Coulthard, M. and Johns, C. (1980) Discourse Intonation and Language Teaching, London, Longman Higher Education.

Cooke, D. (1959) Language of Music, Clarendon paperbacks

Cook, N. (1990) Music, Imagination and Culture, Oxford, Clarendon Press

Frith, S. (1996). Performing Rites: On the Value of Popular Music, Harvard University. Press, Cambridge, Mass

Halliday, M.A.K. (1978) Language as Social Semiotic: The Social Interpretation of Language and Meaning, Baltimore, University Park Press

Kress, G. and van Leeuwen, T. (1996) Reading Images: The Grammar of Visual Design, London, Routledge

Kress, G. (2010) Multimodality, London, Routledge

Levitin, D. (2006) Your Brain on Music, London, Atlantic Books

Machin, D. (2010) Analysing Popular Music, London Routledge.

McConnell- Ginet, S. (1977) 'On Stress and Linguistic Rhythm', Linguistic Inquiry 8, 249336

Middleton, R (1983) 'Play it again, Sam': some notes on the productivity of repetition in popular music'. Popular Music, 3: 215-234 (Cambridge).

O’Toole, M. (1994) The Language of Displayed Art, Rutherford, Fairleigh Dickinson Press

Schafer, R.M. (1977) The Tuning of the World, Toronto, McClelland and Stewart,

Tagg, P. (1982) Nature as a Musical Mood Category, Nordens working paper series. http://www.tagg.org/articles/xpdfs/nature.pdf

Tagg, P. (1984) 'Understanding Musical Time Sense' in Tvarspel - Festskrift for Jan Ling (50 år), Göteborg, Skriften fran Musikvetenskapliga Institutionen http://www.tagg.org/articles/xpdfs/timesens.pdf 
Tagg, P. (1994) From Refrain to Rave: The decline of the figure and the rise of the ground, Popular Music 13/2, 209-222

Walser,R. (1993) Running with the Devil: Power, Gender, and Madness in Heavy Metal Music Hanover, N.H.: Wesleyan University Press 